\title{
Simulation of AIDS Spread Model Based on Cellular Automata
}

\author{
Jiatai Gang ${ }^{1, a}$, Sisi Liu ${ }^{1, b}$, Jian Lu' ${ }^{1, c}$, Zhiwei Zhao ${ }^{2, d}$, and Xinxin Tan ${ }^{1, e, *}$ \\ ${ }^{1}$ College of Information Engineering, Dalian University, Dalian, China \\ ${ }^{2}$ College of Environmental and Chemical Engineering, Dalian University, Dalian, China \\ agjt1960@126.com, ' Izsongsisi@163.com, 'Lujian_dldx@163.com, ${ }^{\mathrm{d}} \mathrm{mrszhaozhiwei@163.com,}$ \\ etanxdl@163.com \\ ${ }^{*}$ Corresponding author
}

Keywords: AIDS Spread Model, Cellular Automata, Bridge Population, Simulation.

\begin{abstract}
This paper establishes an AIDS model based on the spread characteristics in China, spread from high-risk to general population, through bridge population based on cellular automata. Matlab is used to simulate the dynamic performance of the model, the simulated results are consistent with statistics from Estimates for the HIV/AIDS Epidemic in China, which shows the feasibility and effectiveness of the proposed model. Then, a simulation of the effects of bridge population on AIDS spread is done and effective optimized control strategies are offered.
\end{abstract}

\section{Introduction}

AIDS (Acquired Immune Deficiency Syndrome), a syndrome caused by a virus called HIV (Human Immunodeficiency Virus), is an infectious chronic disease with a high fatality rate. By 2013, there are an estimated 70 million people infected with HIV, of which 30,000,000 died [1]. At present, although national AIDS prevalence remains low, China has about 19\% of the world's population [2]; therefore, the Chinese growth rate of AIDS cases will have a huge impact on global AIDS prevalence [3]. From the first diagnosed AIDS case in 1985 to the end of August 2014, China has had 490,000 people living with HIV/AIDS and 149,000 deaths [4]. The spread trend of AIDS in China is not optimistic.

Current popular infectious disease models adopt a dynamic system model based on differential equations; however, due to the complexity of AIDS spread mode and epidemiological characteristics, accurate analytical solutions are hard to achieve by mathematical modeling [5]. With the development of computer technologies, many scholars introduced network dynamics to infectious disease research and cellular automaton was considered as an appropriate simulation method. Gang et al. [6] established a CA-based epidemic model to study the impacts of isolation intervention on infectious disease spread. Yang et al. [7] analyzed the segregation power and AIDS-immune persons, influence on the transmission of the diseases. Gao et al. [8] studied the effects of individual behaviors on AIDS spread considering heterogeneity.

AIDS is mainly transmitted by three methods, blood transmission, sexual contact, and mother-to-child transmission. By the end of 2011, the proportion of sexually transmitted HIV infections arrives at $75.2 \%$. At the same time, the epidemic is spreading from high-risk population to general population through bridge population in China [9]. Therefore, it is of great realistic significance and theoretical value to establish an AIDS spread model consistent with the spread characteristics in China from the perspective of HIV infection containment to general population and enriching knowledge in related fields [10].

According new spread trend of AIDS in China, combined with existing research, this paper established an AIDS spread model from high-risk population to general population through bridge population by the way of heterosexual sex based on cellular automata. The paper also studied the effects of bridge population on AIDS spread, offering decision-making basis for concerned departments in making AIDS control and prevention optimization methods and strategies. 


\section{Model Construction}

\section{Population Definition}

In this paper, AIDS high-risk population refers to those injection drug users who conduct high risk behavior and carries HIV, but have not develop into AIDS. The Bridge population is defined as those who have relationship with the high-risk and the general population. Therefore, the bridge population is easily contracted with HIV and transmits it to others [3,5]. Bridge population comprises of sex workers and their clients in this paper.

\section{AIDS Model}

It is assumed that during the propagation period, the studied population can be classified into three states:

Susceptible(S): healthy and non-infection;

Infection(I): infected, but non-displays any symptoms of AIDS with strong infectiousness; AIDS: displays symptoms of AIDS. The propagation flow chart is shown as Fig. 1.

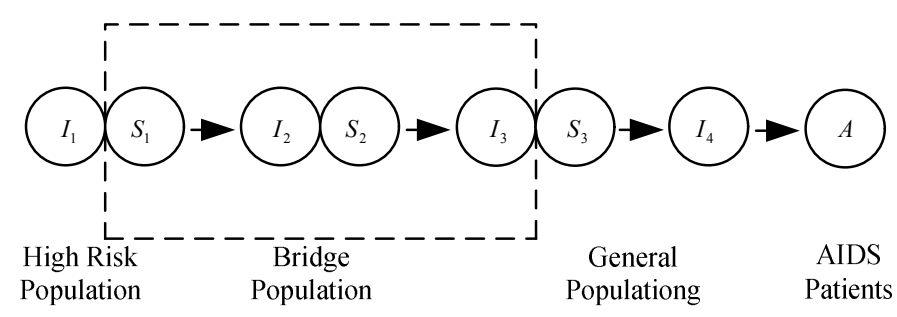

Fig. 1 Propagation flow chart

After sex workers $\left(S_{1}\right)$ have sex (unprotected sexual intercourse) with drug injection users with HIV $\left(I_{1}\right)$, they will become sex workers with HIV $\left(I_{2}\right)$ with a certain infection probability. After clients of sex workers $\left(S_{2}\right)$ have sex with sex workers with HIV $\left(I_{2}\right)$, they will become clients of sex workers with HIV $\left(I_{3}\right)$ with a certain infection probability. After general population $\left(S_{3}\right)$ have sex with clients of sex workers with HIV $\left(I_{3}\right)$, they will become general population with HIV $\left(I_{4}\right)$ with certain infection probability, and then general population with HIV $\left(I_{4}\right)$ will developed into AIDS population $(A)$.

\section{Probability of Infectious}

Sexual transmission through unprotected intercourse is a fundamental HIV spread method from one individual to another. Generally, the higher the frequency of intercourse between two individuals, the higher the likelihood of infection. The probability $P_{C_{i, j} C_{m, n}}^{t}$ of $C_{i, j}$ being infected by neighboring cellular $C_{m, n}$ at time $t$ (year) can by shown as

$$
P_{C_{i, j}}^{t}=\beta C, \quad(m, n) \neq(i, j)
$$

Of which, $\beta$ represents the probability of change from susceptible into HIV infected when in every contact, $C$ represents the average number of coitus per year.

This study takes the total infectiousness of all neighboring cellular of $C_{i, j}$ as $P_{C_{i, j}}^{t}$, the probability of infection at time $t[6]$ :

$$
P_{C_{i, j}}^{t}=\sum_{C_{i, j} \neq C_{m, n}}^{N_{C_{i, j}}} p_{C_{i, j} C_{m, n}}^{t}
$$

Of which, $N_{C_{i, j}}$ represents the set of $C_{i, j}{ }^{\prime} s$ neighbor. 


\section{Rules of Evolution}

For convenience of describing the state characteristics of individuals, $R_{i, j}^{t}=\{0,1,2,3\}$ is used to represent the set of populations of $C_{i, j}$ at time $t$ and 4 numbers are in correlation to the 4 populations: general population, clients of sex workers, female sex workers and high risk population. $S_{i, j}^{t}=\{0,1,2,3,4,5,6\}$ is used to represent the set of states of $C_{i, j}$ at time $t$ and 3 numbers are in correlation to the 3 states S, I and A. The evolution rules for cellular states are as follows:

(1) When $R_{C_{i, j}}^{t}=2$ and $S_{C_{i, j}}^{t}=0$, the individual infection probability $P_{C_{i, j}}^{t}$ of $C_{i, j}$ at time $t$, and use this to determine whether the individual will change to state $S_{i, j}^{t+1}=1$ by $P_{C_{i, j}}^{t}$, or stay susceptible.

(2) When $R_{C_{i, j}}^{t}=1$ and $S_{C_{i, j}}^{t}=0$, the individual infection probability $P_{C_{i, j}}^{t}$ of $C_{i, j}$ at time $t$, and use this to determine whether the individual will change to state $S_{i, j}^{t+1}=1$ by $P_{C_{i, j}}^{t}$, or stay susceptible.

(3) When $R_{C_{i, j}}^{t}=0$ and $S_{C_{i, j}}^{t}=0$, the individual infection probability $P_{C_{i, j}}^{t}$ of $C_{i, j}$ at time $t$, and use this to determine whether the individual will change to state $S_{i, j}^{t+1}=1$ by $P_{C_{i, j}}^{t}$. When the individual is given in probability $P_{C_{i, j}}^{t}$, the individual is changed to $S_{C_{i, j}}^{t+1}=2$, maintained but removed from the transmission process, or stay susceptible.

\section{Simulation and Results}

\section{Parameters Setting}

Let cell size $N=100 \times 100$. Moore's neighborhood is used with radius 1 , unit its' year (y), running time is $25 \mathrm{y}$, and transition probability $p=0.02$. In order to simplify the model, the following assumptions are given.

(1) Birth rate equals mortality rate, that is, the total population remains the same.

(2) There is no related high-risk behavior that would cause HIV/AIDS transmission among individuals of each population.

(3) No other sexual contact exists among heterosexual sex.

Combined with report on the epidemic situation of HIV/AIDS in China and other related study, parameters [11] are set as follows:

Table 1 Parameters description and values in HIV/AIDS Spread Model by heterosexual sex

\begin{tabular}{|c|c|c|}
\hline Parameter & Description & Value \\
\hline$I_{1}^{0}$ & Population size of injection drug users in 2005 & 290,000 \\
\hline$S_{1}^{0}$ & Population size of sex workers in 2005 & $3,650,000$ \\
\hline$S_{2}^{0}$ & Population size of clients of sex workers in 2005 & $28,360,000$ \\
\hline$\beta_{1}$ & $\begin{array}{l}\text { Probability of a male with HIV transmitting to a healthy female per } \\
\text { unprotected sexual intercourse }\end{array}$ & 0.002 \\
\hline$\beta_{2}$ & $\begin{array}{l}\text { Probability of a female with HIV transmitting to a healthy male per } \\
\text { unprotected sexual intercourse }\end{array}$ & 0.001 \\
\hline$C_{1}$ & Average number of sexual intercourses per sex worker in a year & 300 \\
\hline$C_{2}$ & $\begin{array}{l}\text { Average number of sexual intercourses per client of sexual workers in a } \\
\text { year }\end{array}$ & 30 \\
\hline$C_{3}$ & $\begin{array}{l}\text { Average number of sexual intercourses per client of sexual workers with } \\
\text { the fixed sex partner in a year [12] }\end{array}$ & 100 \\
\hline$\omega$ & Conversion rate from HIV to AIDS per year & 0.067 \\
\hline
\end{tabular}




\section{Model Test}

Based on the statistics from A Joint Assessment of HIVIAIDS Prevention, Treatment and Care in China, in 2005, 2007, 2009 and 2010, the median and data interval for HID/AIDS data caused by heterosexual sex are 236,000 (199,000 276,000), 284,000 (223,000 345,000), 328,000 $(248,000 \sim 408,000)$ and 363,000 (288,000 437,000), respectively [13,14,15,16]. Fig. 2 indicates that the data got from the four simulations during 2005 2011 are all fallen into the estimated range. The spread trends in both the estimation and simulation have a good fitting, whose fitting correlation coefficient reaches $99.61 \%$ (0.996149), which shows the proposed model is feasible and effective.

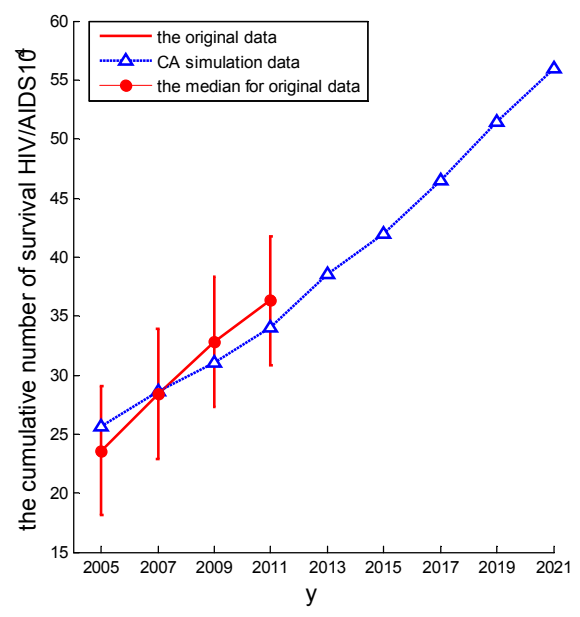

Fig. 2 Comparison between simulated data and estimated data

\section{Effects of bridge population on AIDS spread trend}

AIDS transmission probability stays at 0.0001-0.1 through heterosexual sex [17], which shows that AIDS is less risky than other sexually transmitted diseases. However, due to the existence of other synergy factors which can accelerate HIV transmission, there is a high risk that a HIV carrier without protection measures eventually transmits the virus to others [18]. In China, female sex workers have high HIV infection rate, so, healthy males may transmit HIV virus to their wives or fixed sex partners after unprotected sex intercourses with female sex workers. Therefore, the study on bridge population played a role in controlling HIV/AIDS spread to general population.

Simulation analysis on increasing bridge population. This section will simulate how the bridge population influences AIDS spread to general population increase. Set the cell size $N$ and number of population 1.1 times, twice and 3 times of their initial values and keep other parameters the same.

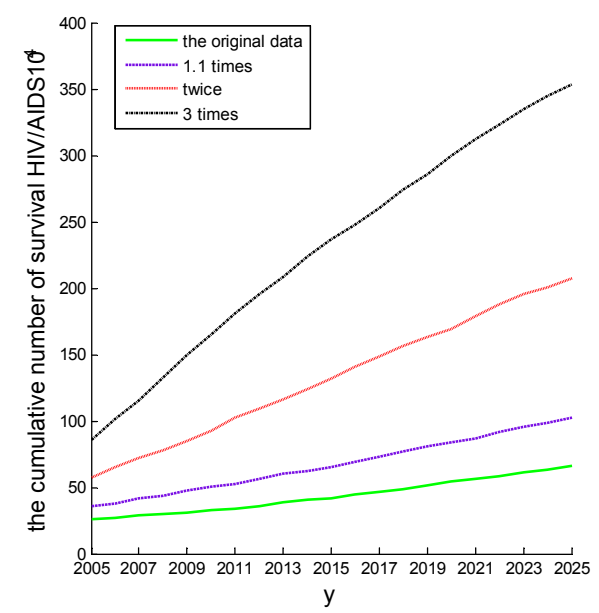

Fig. 3 The increase of bridge population influences AIDS spread to general population 
Seen from Fig. 3, compared with original data, when bridge population size increases by $10 \%$, the cumulative number of HIV/AIDS cases increases significantly to exceed 1 million, and goes up to 1.02 million in 2025 while the original data exceeds 1 million in 2035, which is 10 years in advance. When bridge population size is twice and 3 times of the initial values, the cumulative number of HIV/AIDS cases reaches 1 million in 2011 and 2006, respectively, which are 14 years and 29 years earlier, respectively. The results show that the increase of bridge population will accelerate HIV/AIDS spread to general population.

Simulation analysis on decreasing bridge population. Supposing other parameters remain the same, let

(1)the number of clients of sex workers reduce to $90 \%, 70 \%, 50 \%$ and $30 \%$ of the initial number.

(2)the number of sex workers reduce to $90 \%, 70 \%, 50 \%$ and $30 \%$ of the initial number.

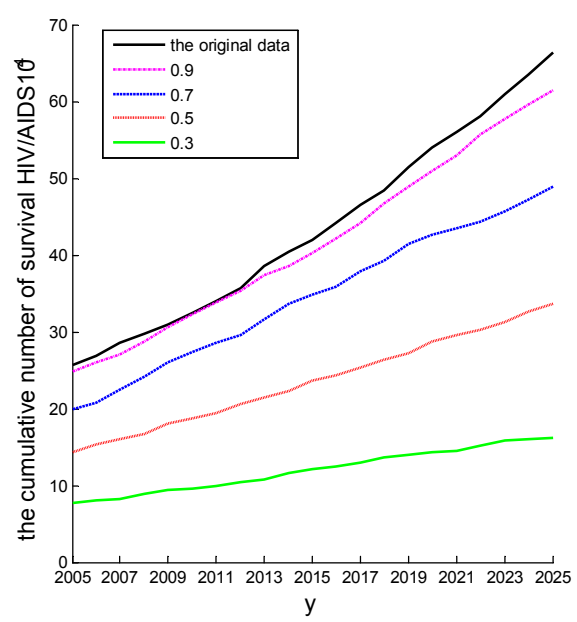

(a) The impact of clients of sex workers on the spread of HIV/AIDS

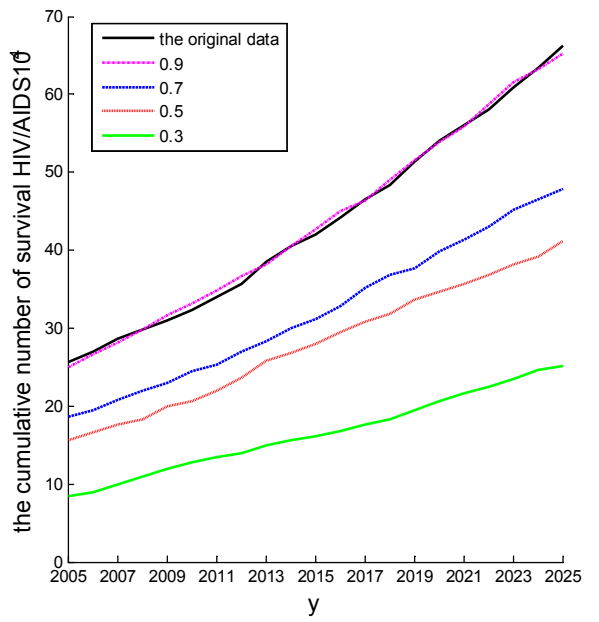

(b) The impact of sex workers on the spread of HIV/AIDS

Fig. 4 The decreas of bridge population influences AIDS spread to general population

As indicated in Fig. 4, with the decrease of the population, the increasing cumulative number of HIV/AIDS cases becomes much less in the same year. From Fig. 4(a), it can be observed that the decline of clients of sex workers will lower HIV/AIDS spread from high-risk population to general population through bridge population. When the number reduces to $30 \%$, the AIDS spread trend from high-risk population to general population through bridge population tends to be stable. From Fig. 4(b), it can be observed that, the AIDS spread from high-risk population to general population through bridge population slows down with the decrease of sex workers. Comparing Fig. 4(a) and Fig. 4(b), it can be found that in the same year, with the same number variation, to decrease clients of sex workers have a greater influence on cumulative number of HIV/AIDS cases than to decrease sex workers.

By performing linear fit on the curves in Fig. 4(a) and 4(b), respectively, we can get the slope of straight line fitting under different population.

Table 2 The slope of straight line fitting under different population

\begin{tabular}{ccccc}
\hline \hline & $90 \%$ & $70 \%$ & $50 \%$ & $30 \%$ \\
\hline sex workers & 2.0469 & 1.5092 & 1.3004 & 0.8277 \\
clients of sex workers & 1.8690 & 1.4692 & 0.9631 & 0.4486 \\
\hline \hline
\end{tabular}

It can be observed in Table 2 that in the same year, with the same population variation, to decrease clients of sex workers have better control effects than to decrease sex workers on AIDS spread from high-risk population to general population through bridge population.

According to the study above, we can see that bridge population has significant effects on AIDS spread. When bridge population increases, the cumulative number of HIV/AIDS cases increases considerably and spread speed from high-risk population to general population through bridge population increases significantly. Otherwise, to decrease bridge population plays a greater pole in 
lowering the cumulative number of HIV/AIDS cases and alleviating the situation of HIV/AIDS spread from high-risk population to general population through bridge population, and under the same conditions, lowering clients of sex workers have the similar effects. Therefore, concerned departments should strengthen the management of bridge population, especially the clients of sex workers, which would better control the HIV/AIDS spread from high-risk population to general population through bridge population.

\section{Conclusions}

Considering the spread characteristics of AIDS in China, this paper establisheds an AIDS spread model from high-risk population to general population through bridge population based on cellular automata. With the use of Matlab simulation, the number of HIV/AIDS infected people through heterosexual sex are derived. Simulation results agree with data from Estimates for the HIV/AIDS Epidemic in China during 2005 2011 with fitting correlation coefficient of 99.61\%. The research shows that the proposed model is feasible and effective. And the effects of bridge population variation on AIDS spread are also simulated.

Meanwhile, the effects of increasing and decreasing bridge population are simulated. When the cell size $N$ and number of population increased 1.1 times, twice and 3 times of their initial values and keep other parameters the same, the results show that the increase of bridge population will accelerate HIV/AIDS spread to general population. When the number of clients of sex workers and sex workers reduce to $90 \%, 70 \%, 50 \%$ and $30 \%$ of the initial number, the results show that the decrease of bridge population will lower HIV/AIDS spread to general population. And it can be found that in the same year to decrease clients of sex workers have a greater influence on cumulative number of HIV/AIDS cases than to decrease sex workers.

Therefore, related departments and governments need to further strengthen the management of bridge population, especially clients of sex workers would have better effects on controlling AIDS spread from high-risk population to general population.

\section{References}

[1] Hongzhou Lu, Qichao Pan, Zhen Ning, A summary of epidemic and control strategy of AIDS in China, Health Education and Health Promotion, 2013, 06:445-448.

[2] QianWang, A comparative study on health resource in China and the whole word, Peking Union Medical College, 2013.

[3] Ning Wang, HIV Epidemic in China and the World: Current Situation and Challenges, Science \& Technology Review, 2005, 23(0507): 4-8.

[4] Information on http://www.chinaaids.cn/yqjc/blbg/201410/t20141028_105842.htm

[5] Zhien Ma, The Mathematical Modeling and Research of Population Ecology, Anhui Jouranl of Education,1996.

Reference to a book:

[6] Jiatai Gang, Qinwu Dai, Shujuan Li, etal, Study of Simulation Method of Vehicle Handling and Stability, Journal of Dalian University, 2014, (3):6-10,18.

[7] Zhifang Pan, Feng Yang, Qinxiao Yang, A Model Research on AIDS Diffusion on Based on Cellular Automaton, Jouranl of Biomedical Engineering, 2011, 03:479-483.

[8] Lu Li, Huiyu Xuan, Baojun Gao etal, CA-based Heterogeneious Epidemic Model for HIV/AIDS Transmission, Journal of Systems Management, 2008, 17(6):704-710.

[9] 2011 Estimates for the HIV/AIDS Epidemic in China, Chinese Journal of AIDS \& STD, 2012,01:1-5. 
[10] Houlin Tang, Fan Lv, Role of Bridge Population in the Transmission of Human Immunodeficiency Virus, Chinese Journal of Epidemiology, 2007, 28(2): 192-194

[11] Junjie Wang, Application of Dynamic Models to Analyze Transmission Characteristic among Five Most-at-Risk Populations in China, Chinese Center For Control And Prevention, 2011.

[12] Lijun Fan, Meiqiong $\mathrm{Wu}$, Zhuozheng Li, Sexual Life of Marride and Incumbency Women:Survey of 63., Chinese Primary Health Care, 2003, 11:50-51.

[13] CMOH, UNAIDS, WHO, eds, 2005 Estimates for the HIV/AIDS Epidemic in China.(2006)

[14] 2007 A Joint Assessment of HIV/AIDS Prevention, Treatment and Care in China: summary, Chinese Nursing Management, 2008, 07:18-19.

[15] CMOH, UNAIDS,WHO,eds, 2009 Estimates for the HIV/AIDS Epidemic in China.(2010)

[16] Powers KA, Poole C, PettiforAE, Cohen MS, Rethinking the heterosexual infeetivity of HIV-1: a systematic review and meta-analysis, The Lancet infectious diseases. 2008;8(9):553-63.

[17] Min Liu, Konglai Zhang, Research Progress of the effect of the spread of HIV through sexual behavior risk factors, Foreign Medical Sciences (Section of Social Medicine), 2003, 01:8-12. 